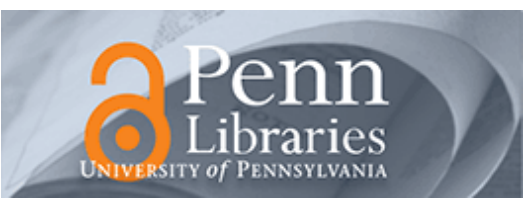

University of Pennsylvania

ScholarlyCommons

March 2002

\title{
Competitively coupled orientation selective cellular neural networks
}

\author{
Bertram E. Shi \\ Hong Kong University of Science and Technology \\ Kwabena A. Boahen \\ University of Pennsylvania, boahen@seas.upenn.edu
}

Follow this and additional works at: https://repository.upenn.edu/be_papers

\section{Recommended Citation}

Shi, B. E., \& Boahen, K. A. (2002). Competitively coupled orientation selective cellular neural networks. Retrieved from https://repository.upenn.edu/be_papers/21

Copyright 2002 IEEE. Reprinted from IEEE Transactions on Circuits and Systems I: Fundamental Theory and Applications, Volume 49, Issue 3, March 2002, pages 388-394.

Publisher URL: http://ieeexplore.ieee.org/xpl/tocresult.jsp?isNumber=21319\&page=1

This material is posted here with permission of the IEEE. Such permission of the IEEE does not in any way imply IEEE endorsement of any of the University of Pennsylvania's products or services. Internal or personal use of this material is permitted. However, permission to reprint/republish this material for advertising or promotional purposes or for creating new collective works for resale or redistribution must be obtained from the IEEE by writing to pubs-permissions@ieee.org. By choosing to view this document, you agree to all provisions of the copyright laws protecting it.

This paper is posted at ScholarlyCommons. https://repository.upenn.edu/be_papers/21

For more information, please contact repository@pobox.upenn.edu. 


\title{
Competitively coupled orientation selective cellular neural networks
}

\author{
Abstract \\ We extend previous work in orientation selective cellular neural networks to include competitive couplings \\ between different layers tuned to different orientations and spatial frequencies. The presence of these \\ interactions sharpens the spatial frequency tuning of the filters in two ways, when compared to a similar \\ architecture proposed previously which lacks these interactions. The first is the introduction of nulls in the \\ frequency response. The second is the introduction of constraints on the passbands of the coupled \\ layers. Based on an understanding of these two effects, we propose a method for choosing spatial \\ frequency tunings of the individual layers to enhance orientation selectivity in the coupled system.

\section{Keywords} \\ cellular neural networks, competition, Gabor filters, image processing, orientation-selective filters \\ Comments \\ Copyright 2002 IEEE. Reprinted from IEEE Transactions on Circuits and Systems I: Fundamental Theory \\ and Applications, Volume 49, Issue 3, March 2002, pages 388-394. \\ Publisher URL: http://ieeexplore.iee. org/xpl/tocresult.jsp?isNumber=21319\&page=1 \\ This material is posted here with permission of the IEEE. Such permission of the IEEE does not in any way \\ imply IEEE endorsement of any of the University of Pennsylvania's products or services. Internal or \\ personal use of this material is permitted. However, permission to reprint/republish this material for \\ advertising or promotional purposes or for creating new collective works for resale or redistribution must \\ be obtained from the IEEE by writing to pubs-permissions@ieee.org. By choosing to view this document, \\ you agree to all provisions of the copyright laws protecting it.
}




\section{Competitively Coupled Orientation Selective Cellular Neural Networks}

\author{
Bertram E. Shi and Kwabena Boahen
}

\begin{abstract}
We extend previous work in orientation selective cellular neural networks to include competitive couplings between different layers tuned to different orientations and spatial frequencies. The presence of these interactions sharpens the spatial frequency tuning of the filters in two ways, when compared to a similar architecture proposed previously which lacks these interactions. The first is the introduction of nulls in the frequency response. The second is the introduction of constraints on the passbands of the coupled layers. Based on an understanding of these two effects, we propose a method for choosing spatial frequency tunings of the individual layers to enhance orientation selectivity in the coupled system.
\end{abstract}

Index Terms-Cellular neural networks, competition, Gabor filters, image processing, orientation-selective filters.

\section{INTRODUCTION}

Cellular neural networks [1] can be used to derive and to analyze circuit architectures implementing orientation selective image filters similar to Gabor filters [2]. The resulting circuit architectures consist of arrays of identical analog continuous time circuits each corresponding to one pixel in the image [3]. Each circuit is interconnected with neighboring circuits in its array. By adjusting the interconnections via external bias voltages, we can tune the array to respond maximally at different scales and orientations.

In this brief, ${ }^{1}$ we extend this work by coupling the outputs of several orientation selective arrays or layers tuned to different scales and orientations so that they compete to model the input. This sharpens spatial frequency tuning. Section II briefly introduces basic concepts in orientation selective filtering with a discussion of the Gabor filter and previous related work. Section III reviews previous work in implementing Gabor-type filters using cellular neural networks. Section IV extends this work to include competitive couplings between several layers tuned to different spatial frequencies, derives closed form expressions for the frequency response, proves that introducing competitive coupling sharpens spatial frequency tuning, and illustrates this via system level simulations. This sharpening is robust in the sense that it exists for any choice of spatial frequency tunings of the individual layers. Section V quantifies the effect of particular choices of spatial frequency tunings by examining the effect of these choices in the passband and stopband of the resulting filters. We introduce a method to choose spatial frequency tunings to enhance orientation tuning. Finally, the conclusion in Section VI describes briefly our approach to the circuit implementation of these cellular neural networks. In particular, because the coupled system is a natural extension of previous work, we can exploit previously developed circuit architectures.

Manuscript received March 13, 2001; revised September 28, 2001. This work was supported in part by the Research Grants Council of Hong Kong, China, under Grant HKUST6218/01E and in part by the U.S. National Science Foundation's LIS/KDI program under Grant KDI-ECS98-74463. This paper was recommended by Associate Editor P. Szolgay.

B. E. Shi is with the Department of Electrical and Electronic Engineering, Hong Kong University of Science and Technology, Kowloon, Hong Kong.

K. Boahen is with the Department of Bioengineering, University of Pennsylvania, Philadelphia, PA 19104 USA.

Publisher Item Identifier S 1057-7122(02)02274-2.

${ }^{1}$ This work was inspired by discussions held at the 2000 Telluride Workshop on Neuromorphic Engineering.

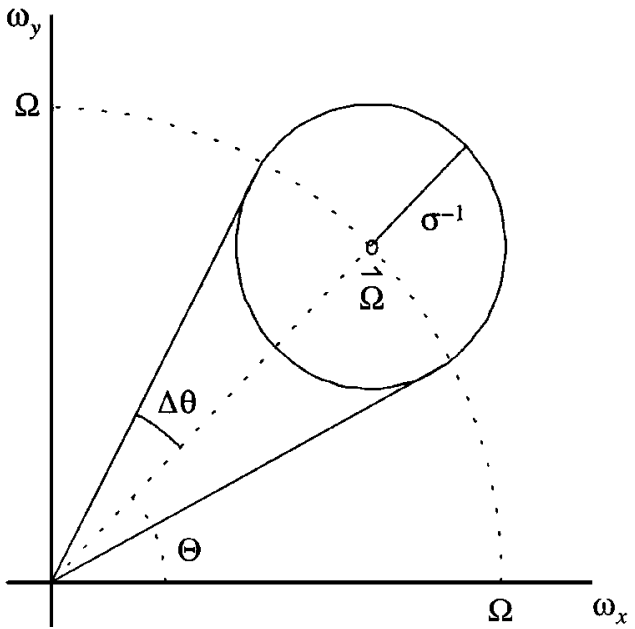

Fig. 1. The solid circle shows the level set where the frequency response of a Gabor filter tuned to $\vec{\Omega}$ with covariance matrix $C=\sigma^{2} I$ drops to $\exp (-0.5)$ of its maximum. The spatial-frequency bandwidth is $2 \sigma^{-1}$. The orientation bandwidth is $2 \triangle \theta$. Note that the lines defining the orientation bandwidth are not tangent to the circle.

\section{ORIENTATION SELECTIVE FILTERING}

A commonly used orientation selective filter is the Gabor filter [4], whose frequency response is a Gaussian centered at a spatial frequency $\vec{\Omega}=\left[\Omega_{x} \Omega_{y}\right]^{T}$ :

$$
G(\vec{\omega})=\exp \left\{-\frac{1}{2}(\vec{\omega}-\vec{\Omega})^{T} C(\vec{\omega}-\vec{\Omega})\right\}
$$

where $\vec{\omega}=\left[\omega_{x} \omega_{y}\right]^{T}$ denotes spatial frequency and $C$ is a positive definite covariance matrix. The Gabor filter has complex valued impulse response

$$
g(\vec{x})=\frac{1}{2 \pi|C|^{1 / 2}} \exp \left\{-\frac{1}{2} \vec{x}^{T} C^{-1} \vec{x}\right\} \exp \left\{j \vec{\Omega}^{T} \vec{x}\right\}
$$

where $\vec{x}=\left[\begin{array}{ll}x & y\end{array}\right]^{T}$ denotes spatial position.

With orientation selective filters, polar coordinates are helpful in describing the spatial frequency domain, as the radius indicates the spatial frequency magnitude and the polar angle indicates the orientation of a sinusoidal grating at frequency $\vec{\omega}$. Thus, the Gabor filter above responds maximally to spatial frequencies with magnitudes near $\Omega=\|\vec{\Omega}\|$ and orientations near $\Theta=\angle \vec{\Omega}$. In the image plane, the orientation corresponds to angle between the $x$ axis and the normal to the level sets of the image intensity.

We define the passband of an orientation selective filter to be the area in the spatial frequency domain where the magnitude of the frequency response is larger than some fraction of the maximum value, and the stopband to be its complement. For the Gabor filter, a convenient fraction is $\exp (-0.5) \approx 0.6$, since the boundary between the passband and stopband is an ellipse centered at $\vec{\Omega}$ whose shape is controlled by the covariance matrix $C$. If $C=\sigma^{2} I$ where $I$ is the identity matrix, the contour is a circle with radius $\sigma^{-1}$.

We define the spatial frequency-magnitude bandwidth of an orientation selective filter to be the distance between the two points along the line $\theta=\Theta$ which intersect the boundary of the passband. We define the orientation bandwidth to be the difference between the angles at which a circle centered at the origin with radius $\Omega$ intersects the boundary. For a Gabor filter with circularly symmetric frequency response, the spatial frequency magnitude bandwidth is $2 \sigma^{-1}$ and the orientation bandwidth is $2 \triangle \theta=4 \sin ^{-1}\left((2 \sigma \Omega)^{-1}\right)$. See Fig. 1 . 
The implementation of orientation selective filters using neuromorphic concepts has been the focus of much related work. For an overview, see [5]. Most of this work has not incorporated competitive interconnections between orientation selective outputs. However, one notable exception is work on analog VLSI implementations of the Boundary Contour System [6], [7].

\section{UNCOUPLED ORIENTATION SELECTIVE LAYERS}

In this section, we briefly review the previous work in orientation selective cellular neural networks which the current work is based upon [2]. This paper focuses on the system level description and the computational properties of the architecture. For information regarding circuit design and implementation, please refer to [3].

Suppose we wish to filter a real valued image $u(m, n)$ with $K$ filters tuned to center frequencies $\vec{\Omega}_{k}$ for $k=0, \ldots, K-1$. Let the complex valued output of each filter, $\vec{\nu}_{k}=\left\{\nu_{k}(m, n)\right\}$, be the values that minimize the cost function

$$
\begin{aligned}
& E_{k}\left(\vec{\nu}_{k}\right) \\
& =\frac{1}{2} \sum_{m, n}\left\|\nu_{k}(m, n)-u(m, n)\right\|^{2} \\
& +\frac{1}{2\left(\triangle \Omega_{x k}\right)^{2}} \sum_{m, n}\left\|\nu_{k}(m, n)-e^{-j \Omega_{x k}} \nu_{k}(m+1, n)\right\|^{2} \\
& +\frac{1}{2\left(\triangle \Omega_{y k}\right)^{2}} \sum_{m, n}\left\|\nu_{k}(m, n)-e^{-j \Omega_{y k}} \nu_{k}(m, n+1)\right\| \|^{2} .
\end{aligned}
$$

This cost function is the sum of two terms: a data fidelity term that penalizes the difference between the filter output and input and a regularization term that is minimized if the output is a complex exponential waveform with spatial frequency $\vec{\Omega}_{k}$. The amount each term contributes to the cost function is controlled by the parameters $\vec{\triangle} \Omega_{k}=$ $\left(\triangle \Omega_{x k}, \triangle \Omega_{y k}\right)$, which control the filter bandwidth.

Differentiating this cost function with respect to the real and imaginary parts of $\nu_{k}(m, n)$, we obtain two equations that can be compactly expressed as the real and imaginary parts of

$$
\begin{aligned}
\frac{d E\left(\vec{\nu}_{k}\right)}{d \nu_{k}(m, n)}= & \left(1+\frac{2}{\left(\triangle \Omega_{x k}\right)^{2}}+\frac{2}{\left(\triangle \Omega_{y k}\right)^{2}}\right) \nu_{k}(m, n) \\
& -\frac{e^{j \Omega_{x k}}}{\left(\triangle \Omega_{x k}\right)^{2}} \nu_{k}(m-1, n) \\
& -\frac{e^{-j \Omega_{x k}}}{\left(\triangle \Omega_{x k}\right)^{2}} \nu_{k}(m+1, n) \\
& -\frac{e^{j \Omega_{y k}}}{\left(\triangle \Omega_{y k}\right)^{2}} \nu_{k}(m, n-1) \\
& -\frac{e^{-j \Omega_{y k}}}{\left(\triangle \Omega_{y k}\right)^{2}} \nu_{k}(m, n+1)-u(m, n)
\end{aligned}
$$

where $d E\left(\vec{\nu}_{k}\right) / d \nu_{k}(m, n)=\left(d E\left(\vec{\nu}_{k}\right) / d \operatorname{Re}\left\{\nu_{k}(m, n)\right\}\right)+$ $j\left(d E\left(\vec{\nu}_{k}\right) / d \operatorname{Im}\left\{\nu_{k}(m, n)\right\}\right)$. If we set $d \nu_{k}(m, n) / d t=$ $-\left(d E\left(\vec{\nu}_{k}\right) / d \nu_{k}(m, n)\right)$ for all $(m, n)$, we obtain a set of coupled differential equations that minimizes the cost function along its trajectory. These differential equations are that of a single layer cellular neural network with complex valued state

$$
\begin{array}{r}
\frac{d \nu_{k}(m, n)}{d t}=-\nu_{k}(m, n)+\sum_{o, p} a_{k k}(o, p) f\left(\nu_{k}(m+o, n+p)\right) \\
+b_{k}(0,0) u(m, n)
\end{array}
$$

where the output nonlinearity $f(x)=x$ and the feedback and feedforward cloning templates are

$$
\begin{aligned}
& A_{k k}=\left[\begin{array}{ccc}
a_{k k}(-1,1) & a_{k k}(0,1) & a_{k k}(1,1) \\
a_{k k}(-1,0) & a_{k k}(0,0) & a_{k k}(1,0) \\
a_{k k}(-1,-1) & a_{k k}(0,-1) & a_{k k}(1,-1)
\end{array}\right] \\
& =\left[\begin{array}{ccc}
0 & \frac{e^{-j \Omega_{y k}}}{\left(\triangle \Omega_{y k}\right)^{2}} & 0 \\
\frac{e^{j \Omega_{x k}}}{\left(\triangle \Omega_{x k}\right)^{2}} & {\left[-\frac{2}{\left(\triangle \Omega_{x k}\right)^{2}}-\frac{2}{\left(\triangle \Omega_{y k}\right)^{2}}\right]} & \frac{e^{-j \Omega_{x k}}}{\left(\triangle \Omega_{x k}\right)^{2}} \\
0 & \frac{e^{j \Omega_{y k}}}{\left(\triangle \Omega_{y k}\right)^{2}} & 0
\end{array}\right]
\end{aligned}
$$

and $B_{k k}=\left[b_{k k}(0,0)\right]=[1]$. We refer to the each set of outputs $\vec{\nu}_{k}=\left\{\nu_{k}(m, n)\right\}$ as one layer.

If $u(m, n)$ is constant in time, then $\nu_{k}(m, n)$ at steady state is the desired filter output. The convexity of the cost function guarantees stability of the cellular neural network. To find the spatial frequency response of each filter, assume an infinite array and define $U(\vec{\omega})$ to be the discrete space Fourier transform of $u(m, n)$

$$
U(\vec{\omega})=\sum_{n=-\infty}^{\infty} \sum_{m=-\infty}^{\infty} u(m, n) e^{-j \omega_{x} m-j \omega_{y} n}
$$

and $V_{k}(\vec{\omega})$ to be the discrete space Fourier transform of $\nu_{k}(m, n)$. Setting $d \nu_{k}(m, n) / d t=0$ in (2) and taking Fourier transforms

$$
H_{k}^{u}(\vec{\omega})=\frac{V_{k}(\vec{\omega})}{U(\vec{\omega})}=\frac{1}{1+D_{k}(\vec{\omega})}
$$

where

$$
D_{k}(\vec{\omega})=\frac{2-2 \cos \left(\omega_{x}-\Omega_{x k}\right)}{\left(\triangle \Omega_{x k}\right)^{2}}+\frac{2-2 \cos \left(\omega_{y}-\Omega_{y k}\right)}{\left(\triangle \Omega_{y k}\right)^{2}} .
$$

The superscript " $u$ " is used to indicate that this is the transfer function for the uncoupled array.

For the Gabor filter we defined the passband to be the area where the frequency response is greater than $\exp (-0.5) \approx 0.6$ of its maximum value. For these filters, we choose to define the passband to be the area where the frequency response is greater than half its maximum value $(-6 \mathrm{~dB})$, i.e., $D_{k}(\vec{\omega})>1$. This leads to expressions for the contour defining the passband and for the orientation and spatial frequency bandwidth which are similar to those found for the Gabor filter. For $\vec{\omega}$ close to $\bar{\Omega}$,

$$
D_{k}(\vec{\omega}) \approx \frac{\left(\omega_{x}-\Omega_{x k}\right)^{2}}{\left(\triangle \Omega_{x k}\right)^{2}}+\frac{\left(\omega_{y}-\Omega_{y k}\right)^{2}}{\left(\triangle \Omega_{y k}\right)^{2}} .
$$

Thus, the 6-dB contour is approximately an ellipse centered at $\vec{\Omega}$. Unlike the Gabor filter where the corresponding ellipse can be rotated and scaled arbitrarily, the major and minor axes of this ellipse are always parallel to the $\omega_{x}$ and $\omega_{y}$ axes. Only their lengths $2 \triangle \Omega_{x k}$ and $2 \triangle \Omega_{y k}$ can be controlled. Define

$$
Q_{k}=\sqrt{\left(\frac{\Omega_{x k}}{2 \triangle \Omega_{x k}}\right)^{2}+\left(\frac{\Omega_{y k}}{2 \triangle \Omega_{y k}}\right)^{2}}
$$

to be the quality factor, characterizing the sharpness of the tuning. If $\triangle \Omega_{x k}=\triangle \Omega_{y k}=\triangle \Omega_{k}$, the 6-dB contour is approximately circular with radius $\triangle \Omega_{k}$ and $Q_{k}=\Omega_{k} /\left(2 \triangle \Omega_{k}\right)$. Referring to Fig. 1, the spatial frequency magnitude bandwidth is $2 \triangle \Omega_{k}$ and the orientation bandwidth is

$$
2 \triangle \theta=4 \sin ^{-1}\left(\frac{\triangle \Omega_{k}}{2 \Omega_{k}}\right)
$$




\section{COMPETITIVEly COUPLED LAYERS}

In this section, we extend the previous architecture to allow outputs of layers tuned to different center frequencies to interact so that their sum best models the input. This approach is similar to that proposed by Daugman [8]. We derive closed form expressions for the frequency responses of the layers and use these expressions to prove that introducing competition, enhances spatial frequency selectivity.

Suppose we wish to filter a real valued image $u(m, n)$ at with $K$ filters tuned to center frequencies $\vec{\Omega}_{k}$ for $k=0, \ldots, K-1$. Let the filter outputs $\vec{\nu}=\left\{\vec{\nu}_{1}, \vec{\nu}_{2}, \ldots, \vec{\nu}_{K}\right\}$ be the values that collectively minimize the single cost function

$$
\begin{aligned}
& E(\vec{\nu}) \\
& =\frac{1}{2} \sum_{m} \sum_{n}\left\|\sum_{k} \nu_{k}(m, n)-u(m, n)\right\|^{2} \\
& +\frac{1}{2} \sum_{k} \frac{\sum_{m} \sum_{n}\left\|\nu_{k}(m, n)-e^{-j \Omega_{x k}} \nu_{k}(m+1, n)\right\|^{2}}{\left(\triangle \Omega_{x k}\right)^{2}} \\
& +\frac{1}{2} \sum_{k} \frac{\sum_{m} \sum_{n}\left\|\nu_{k}(m, n)-e^{-j \Omega_{y k}} \nu_{k}(m, n+1)\right\|^{2}}{\left(\triangle \Omega_{y k}\right)^{2}}
\end{aligned}
$$

which combines the $K$ separate cost functions (1) into one. The critical difference is the data fidelity term. In (1), the data fidelity term penalizes the difference between the input image and a single output. In (6), the data fidelity term penalizes the difference between the input image and the sum of the outputs. This introduces competitive couplings between the different orientations.

As in Section III, we differentiate the cost function with respect to the real and imaginary parts of $\nu_{k}(m, n)$, set the result equal to $-d \nu_{k}(m, n) / d t$ and obtain the equations for a multi-layer cellular neural network with complex valued state

$$
\begin{array}{r}
\frac{d \nu_{k}(m, n)}{d t}=-\nu_{k}(m, n) \sum_{l, o, p} a_{k l}(o, p) f\left(\nu_{k+l}(m+o, n+p)\right) \\
+b_{k}(0,0) u(m, n)
\end{array}
$$

where the output nonlinearity, the feedforward template $B_{k}$ and intralayer feedback template $A_{k k}$ are the same as in Section III, and the inter-layer feedback templates $A_{k l}$ for $l \neq k$ are given by

$$
A_{k l}=\left[a_{k l}(0,0)\right]=[-1] .
$$

Since the $A_{k l}$ are negative, the inter-layer connections are competitive, since a large response from layer $l$ will inhibit the response from layer $k$. If we eliminate the coupling between layers by setting the $A_{k l}=$ 0 , the CNN equation for the coupled system (7) reduces to the CNN equation for the uncoupled system (2). We thus refer to the parameters $\vec{\Omega}_{k}$ and $\vec{\triangle} \Omega_{k}$ as the uncoupled tunings of the coupled layers.

\section{A. Frequency Response}

Since (7) is linear, we can find the frequency response of each array as in Section III. In the following, we assume that center frequencies $\vec{\Omega}_{k}$ are distinct modulo $2 \pi$.

Taking the Fourier transform of (7)

$$
U(\vec{\omega})=\sum_{f} V_{j}(\vec{\omega})+D_{k}(\vec{\omega}) V_{k}(\vec{\omega})
$$

where $D_{k}(\vec{\omega})$ is given in (4). Note that $D_{k}(\vec{\omega}) \geq 0$ with equality if and only if $\vec{\omega}=\vec{\Omega}$ modulo $2 \pi$. There are two cases which we must consider:

Case 1) $\vec{\omega} \neq \vec{\Omega}_{k}$ for all $k$.
In this case, $D_{k}(\vec{\omega})>0$ for all $k$. Equation (8) can be solved by noting that for all $k$,

$$
V_{k}(\vec{\omega})=\frac{U(\vec{\omega})-\sum_{j} V_{j}(\vec{\omega})}{D_{k}(\vec{\omega})} .
$$

Summing over $k$

$$
\sum_{k} V_{k}(\vec{\omega})=\left\{U(\vec{\omega})-\sum_{j} V_{j}(\vec{\omega})\right\} \sum_{k} D_{k}(\vec{\omega})^{-1} .
$$

Thus

$$
\sum_{k} V_{k}(\vec{\omega})=\frac{\sum_{k} D_{k}(\vec{\omega})^{-1}}{1+\sum_{k} D_{k}(\vec{\omega})^{-1}} U(\vec{\omega}) .
$$

Substituting into (9)

$$
H_{k}^{c}(\vec{\omega})=\frac{V_{k}(\vec{\omega})}{U(\vec{\omega})}=\frac{1}{1+D_{k}(\vec{\omega})+\sum_{j \neq k} \frac{D_{k}(\vec{\omega})}{D_{j}(\vec{\omega})}} .
$$

The superscript " $c$ " is used to indicate that this is the transfer function for the coupled array.

Case 2) $\vec{\omega}=\vec{\Omega}_{l}$ for some $l$.

Since $D_{l}(\vec{\omega})=0$, evaluating (8) for $k=l$

$$
\sum_{j} V_{j}(\vec{\omega})=U(\vec{\omega})
$$

For $k \neq l, D_{k}(\vec{\omega})>0$. By (9), $V_{k}(\vec{\omega})=0$. Substituting into (11), $V_{l}(\vec{\omega})=U(\vec{\omega})$. Thus

$$
H_{k}^{c}(\vec{\omega})=\frac{V_{k}(\vec{\omega})}{U(\vec{\omega})}=\delta(k-l)
$$

where $\delta(k)$ denotes the discrete impulse. This expression is the limit of (10) as $D_{l}(\vec{\omega}) \rightarrow 0$.

Equation (12) shows that when the input spatial frequency component is exactly equal to tuned spatial frequency of one of the arrays, that spatial frequency is passed directly by the corresponding array and completely blocked by all others, independent of the uncoupled bandwidths. Note also that the competitive couplings do not modify the spatial frequency to which each layer responds maximally.

\section{B. Enhancement of Spatial Frequency Tuning}

The coupled system should exhibit a sharper spatial frequency tuning in comparison with the uncoupled system. To see this intuitively, assume that the arrays are tuned to the same spatial frequency magnitude and orientations $\theta_{0}<\theta_{1}<\cdots<\theta_{K-1}$. Consider an input consisting of two lines oriented at angles $\theta_{1}$ and $\theta_{3}$ which intersect at the origin. In the uncoupled case, there will be a strong response at the array tuned to the orientation $\theta_{2}$ which lies between $\theta_{1}$ and $\theta_{3}$, since both lines will contribute to the output. On the other hand, in the coupled case, large responses at the outputs tuned to $\theta_{1}$ and $\theta_{3}$ will suppress the output tuned to the orientation $\theta_{2}$.

The following theorem justifies this intuition mathematically. We compare the frequency responses of coupled and uncoupled layers with the same uncoupled tunings. Suppose the response of the $k$ th layer to a sine wave grating with frequency $\vec{\omega}$ is smaller than the response of the $l$ th array. The ratio

$$
\frac{H_{k}(\vec{\omega})}{H_{l}(\vec{\omega})}<1
$$

measures the sharpness of the tuning. A smaller ratio indicates sharper tuning, since the response of the $k$ th array is more suppressed. 
Proposition 1: Consider one coupled set and one uncoupled set of orientation selective arrays tuned to $K$ distinct spatial frequencies $\left\{\vec{\Omega}_{k}\right\}_{k=1}^{K}$. Define $H_{k}^{c}(\vec{\omega})$ and $H_{k}^{u}(\vec{\omega})$ to be the transfer functions from the input to the output tuned to spatial frequency $\vec{\Omega}_{k}$ for the coupled and the uncoupled array. If for some $k, l$ and $\vec{\omega}$

$$
\frac{H_{k}^{u}(\vec{\omega})}{H_{l}^{u}(\vec{\omega})}<1
$$

then

$$
\frac{H_{k}^{c}(\vec{\omega})}{H_{l}^{c}(\vec{\omega})}<\frac{H_{k}^{u}(\vec{\omega})}{H_{l}^{u}(\vec{\omega})} .
$$

Proof: For the uncoupled arrays, we have from (3)

$$
\frac{H_{k}^{u}(\vec{\omega})}{H_{l}^{u}(\vec{\omega})}=\frac{1+D_{l}(\vec{\omega})}{1+D_{k}(\vec{\omega})} .
$$

If $D_{l}(\vec{\omega})=0$, then by (12)

$$
\frac{H_{k}^{c}(\vec{\omega})}{H_{l}^{c}(\vec{\omega})}=0<\frac{H_{k}^{u}(\vec{\omega})}{H_{l}^{u}(\vec{\omega})} .
$$

Otherwise, (13) implies that $0<D_{l}(\vec{\omega})<D_{k}(\vec{\omega})$

$$
\begin{aligned}
& D_{l}(\vec{\omega})+D_{k}(\vec{\omega}) D_{l}(\vec{\omega})<D_{k}(\vec{\omega})+D_{k}(\vec{\omega}) D_{l}(\vec{\omega}) \\
& D_{l}(\vec{\omega})\left(1+D_{k}(\vec{\omega})\right)<D_{k}(\vec{\omega})\left(1+D_{l}(\vec{\omega})\right) \\
& \frac{H_{k}^{c}(\vec{\omega})}{H_{l}^{c}(\overrightarrow{\bar{\omega}})}=\frac{D_{l}(\vec{\omega})}{D_{k}(\overrightarrow{\bar{\omega}})}<\frac{1+D_{l}(\vec{\omega})}{1+D_{k}(\overrightarrow{\bar{\omega}})}=\frac{H_{k}^{u}(\vec{\omega})}{H_{l}^{u}(\vec{\omega})}
\end{aligned}
$$

where the left hand equality follows from (10).

Introducing coupling between layers also modifies the temporal response of the layers. In the uncoupled system, each spatial frequency component evolves independently. Defining $\vec{V}^{u}(\vec{\omega})=\left[V_{k}^{u}(\vec{\omega})\right]_{k=0}^{K-1}$ to be the vector of spatial frequency components at frequency $\vec{\omega}$ across the layers

$$
\frac{d}{d t} \vec{V}^{u}(\vec{\omega})=-A^{u}(\vec{\omega}) \cdot \vec{V}^{u}(\vec{\omega})+U(\vec{\omega}) \cdot \overrightarrow{1}
$$

where $A^{u}(\vec{\omega})=I+\operatorname{diag}\left\{D_{k}(\vec{\omega})\right\}$ is a $K \times K$ diagonal matrix, $I$ is the $K \times K$ identity matrix and $\overrightarrow{1}$ is a $K$ dimensional vector of ones. Since $A^{u}(\vec{\omega})$ is diagonal, the spatial frequency component at $\vec{\omega}$ evolves independently from layer to layer. In the coupled system, each spatial frequency component also evolves independently, but they are coupled across layers. Denoting $\vec{V}^{c}(\vec{\omega})=\left[V_{k}^{c}(\vec{\omega})\right]_{k=0}^{K-1}$

$$
\frac{d}{d t} \vec{V}^{c}(\vec{\omega})=-A^{c}(\vec{\omega}) \cdot \vec{V}^{c}(\vec{\omega})+U(\vec{\omega})
$$

where $A^{c}(\vec{\omega})=\overrightarrow{1} \cdot \overrightarrow{1}^{T}+\operatorname{diag}\left\{D_{k}(\vec{\omega})\right\}$ is a $K \times K$ matrix with the same diagonal entries as $A^{u}(\vec{\omega})$, but ones instead of zeros in the off diagonal entries. To compare the temporal evolution of the coupled and uncoupled systems, we can compare the minimum eigenvalues of $A^{u}(\vec{\omega})$ and $A^{c}(\vec{\omega})$. Since the time constants are the reciprocal of the eigenvalues, the smaller the eigenvalue, the slower the time constant. Fig. 2 plots the minimum eigenvalues for a set of three layers through a cross section of the $\vec{\omega}$ plane. The minimum eigenvalue of the coupled system is smaller than that of the uncoupled system. Thus, the sharpened spatial orientation selectivity comes at the cost of a slower temporal response.

\section{DESIGN CRITERIA}

The sharpening exhibited by this system is robust in the sense that the theorem above does not depend upon any conditions on the selection of

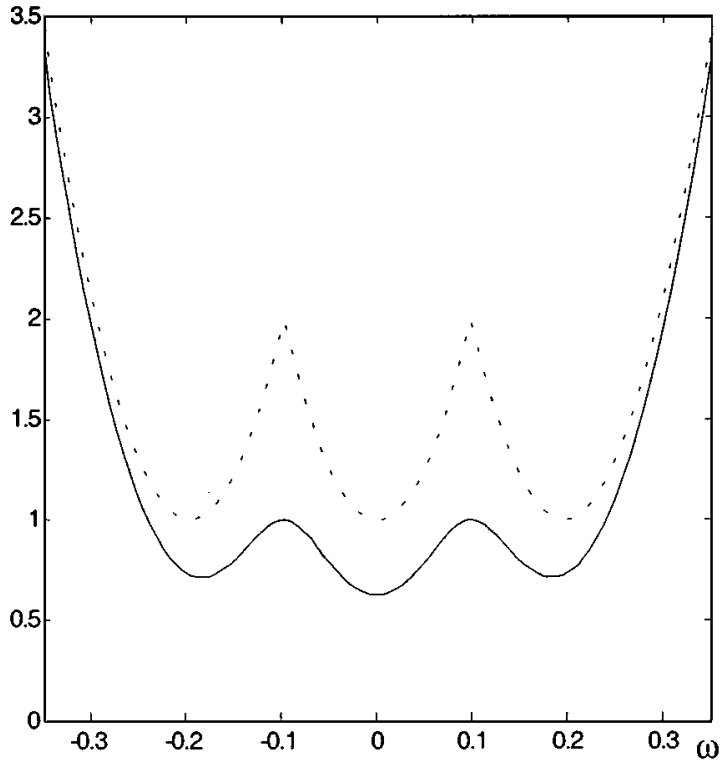

Fig. 2. Minimum eigenvalues of the matrices $A^{u}(\vec{\omega})$ (dotted line) and $A^{c}(\vec{\omega})$ (solid line) plotted versus $\omega$ for the cross section $\vec{\omega}=(\omega, 0)$. The system contains three layers tuned to spatial frequencies $(\pi / 16,0),(0,0)$ and $(-\pi / 16,0)$ with equal uncoupled bandwidths corresponding to $Q=1$ for the nonzero spatial frequencies.

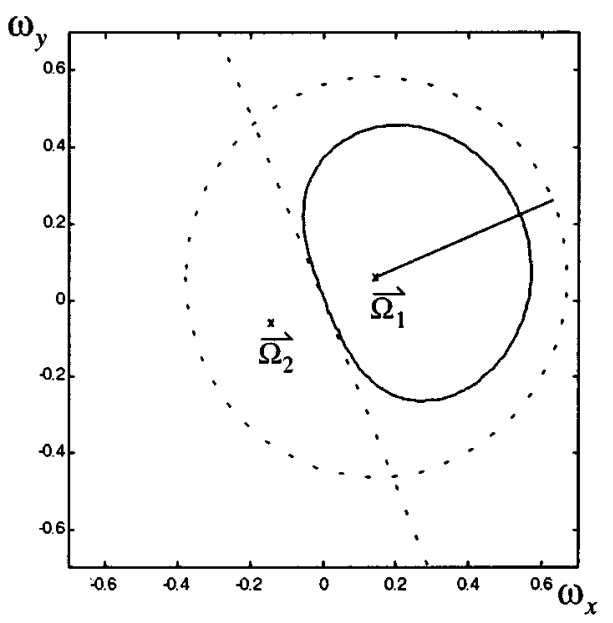

Fig. 3. Constraints on the passband for an array tuned to $\vec{\Omega}_{1}$ introduced by a second coupled layer tuned to $\vec{\Omega}_{2}$. The two coupled layers tuned to equal spatial frequency magnitude $\Omega=\pi / 20$, equal uncoupled sharpness $Q=0.15$ and orientations $\theta_{1}=\pi / 8$ and $\theta_{2}=-\pi / 8$. The solid line shows the actual 6 $\mathrm{dB}$ contour. The dashed circle shows the boundary of the constraint (14). The dashed straight line shows the boundary of the constraint (15).

tuned spatial frequencies or in the bandwidths of the individual arrays, apart from the requirement that the tunings of the different arrays be distinct. However, the amount and nature of the sharpening will depend critically on how the center frequencies and bandwidths are chosen. Two effects of the competitive couplings are helpful in choosing appropriate tunings. The first concerns stopband of the filters. As pointed out in Section IV, an array tuned to $\vec{\Omega}_{k}$ introduces a null in the frequency responses of the other arrays at $\vec{\Omega}_{k}$. The second concerns the passband of the filters. Competitive couplings introduce constraints on the passbands, as discussed below.

Assume that all arrays are tuned so that their frequency are approximately circularly symmetric, i.e.,

$$
\triangle \Omega_{k}=\triangle \Omega_{x k}=\triangle \Omega_{y k} \text { for all } k .
$$




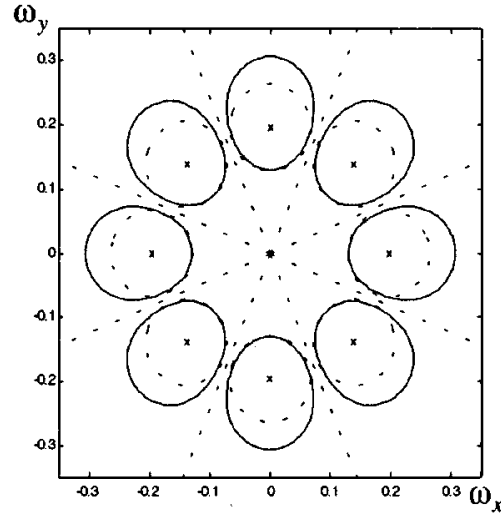

(a)

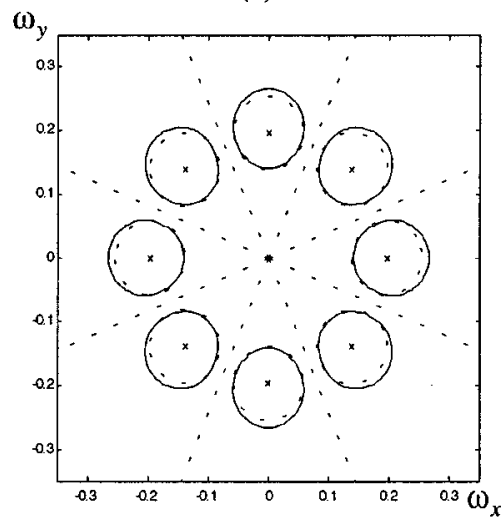

(d)

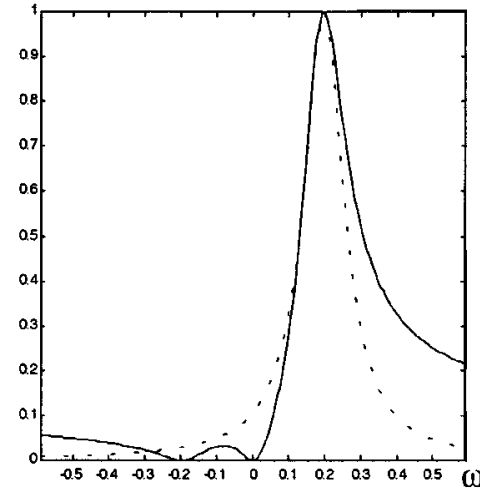

(b)

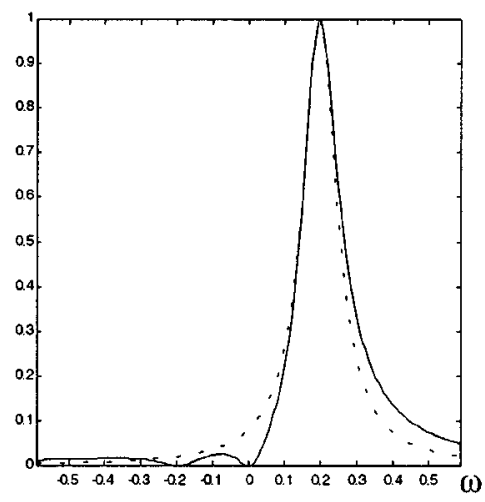

(e)

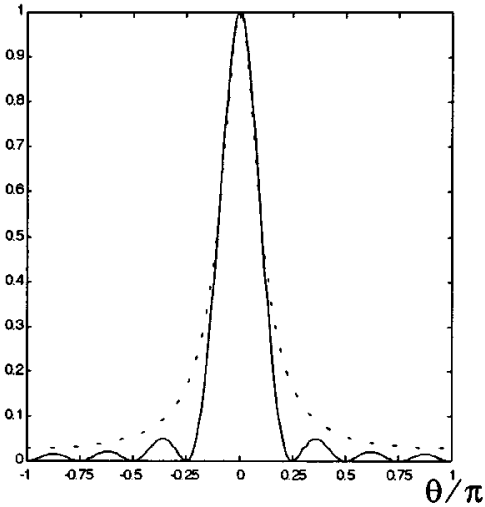

(c)

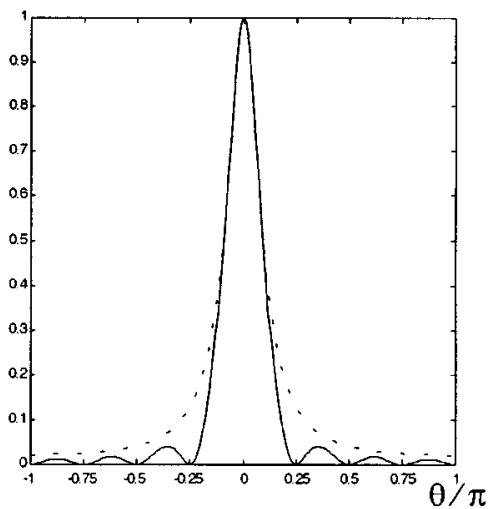

(f)

Fig. 4. Frequency responses from a set of nine coupled layers. (a) Solid circles show 6-dB contours of the frequency response for the eight orientation selective layers in the coupled system. Each layer has an uncoupled $Q=0.1$. Dashed circles show 6-dB contours for uncoupled layers tuned to the same center frequency and orientation bandwidth $(Q=1.46)$. Dashed radial lines indicate the constraints introduced by (15). (b) Frequency responses of the coupled (solid) and uncoupled (dashed) layers tuned to $\theta=0$ plotted versus spatial frequency magnitude $\omega$ along a radial line $\theta=0$. (c) Frequency responses of the coupled (solid) and uncoupled (dashed) layers tuned to $\theta=0$ plotted versus orientation angle along a circle with radius $\Omega$. (d)-(f) Similar plots as above, except each layer in the coupled system has an uncoupled $Q=1.0$ and each layer in the uncoupled system has $Q=1.69$.

We begin with the simplest case of two competing arrays tuned to spatial frequencies, $\vec{\Omega}_{1}$ and $\vec{\Omega}_{2}$. For $\vec{\omega} \neq \vec{\Omega}_{2}$, the first array has transfer function

$$
H_{1}^{c}(\vec{\omega})=\frac{1}{1+D_{1}(\vec{\omega})+\frac{D_{1}(\vec{\omega})}{D_{2}(\vec{\omega})}} .
$$

Since $D_{1}(\vec{\omega}) \geq 0$ and $D_{2}(\vec{\omega}) \geq 0$, a necessary condition for $H_{1}^{c}(\vec{\omega})>1 / 2$ is that both

$$
D_{1}(\vec{\omega})<1
$$

and

$$
D_{1}(\vec{\omega})<D_{2}(\vec{\omega})
$$

Assuming the approximation in (5) is exact, constraint (14) is satisfied for all $\vec{\omega}$ within a circle of radius $\triangle \Omega_{k}$ centered at $\vec{\Omega}_{1}$. Since this circle is the $6 \mathrm{~dB}$ contour in the uncoupled case, this constraint also illustrates the orientation sharpening by the coupled system. The boundary of constraint (15) is a circle with center

$$
\vec{\Omega}_{12}=w_{1} \vec{\Omega}_{1}+w_{2} \vec{\Omega}_{2}
$$

where

$$
w_{1}=\frac{\left(\triangle \Omega_{2}\right)^{2}}{\left(\triangle \Omega_{2}\right)^{2}-\left(\triangle \Omega_{1}\right)^{2}} \quad w_{2}=\frac{-\left(\triangle \Omega_{1}\right)^{2}}{\left(\triangle \Omega_{2}\right)^{2}-\left(\triangle \Omega_{1}\right)^{2}} .
$$

Since $w_{1}+w_{2}=1$, the center lies on the line connecting $\vec{\Omega}_{1}$ and $\vec{\Omega}_{2}$. The radius is the geometric mean of the distances from $\vec{\Omega}_{1}$ and $\vec{\Omega}_{2}$ to the center

$$
R_{12}=\sqrt{\left\|\vec{\Omega}_{1}-\vec{\Omega}_{12}\right\|\left\|\vec{\Omega}_{2}-\vec{\Omega}_{12}\right\|} .
$$

For $\triangle \Omega_{1}<\triangle \Omega_{2}$, the circle contains $\vec{\Omega}_{1}$ but not $\vec{\Omega}_{2}$ and the $6 \mathrm{~dB}$ contour lies within the circle. For $\triangle \Omega_{1}>\triangle \Omega_{2}$, the circle contains $\vec{\Omega}_{2}$ but not $\vec{\Omega}_{1}$ and the $6 \mathrm{~dB}$ contour lies outside the circle. For $\triangle \Omega_{1}=$ $\triangle \Omega_{2}$, the circle reduces to the line bisecting the line segment from $\vec{\Omega}_{1}$ to $\vec{\Omega}_{2}$ perpendicularly. See Fig. 3 .

Each additional layer adds another constraint on the 6- $\mathrm{dB}$ contour whose position and size depends upon its uncoupled tuning. Competing layers whose center frequencies are closest to the center frequency of a layer will have the greatest effect on its spatial tuning.

To enhance orientation tuning, we couple $K+1$ layers. The first $K$ layers are tuned to the same spatial frequency magnitude $\Omega$, the same uncoupled bandwidths, but different orientations spaced equally between 0 and $2 \pi$. In other words,

$$
\Omega_{x k}=\Omega \cos \left(\theta_{k}\right) \quad \Omega_{y k}=\Omega \sin \left(\theta_{k}\right)
$$

where $\theta_{k}=2 \pi k / K$ for $k=0,1, \ldots, K-1$. The final layer is tuned to spatial DC with the same uncoupled bandwidth as the first $K$ layers. For each layer $k$, adjacent orientations $\theta_{k-1}$ and $\theta_{k+1}$ constrain the 


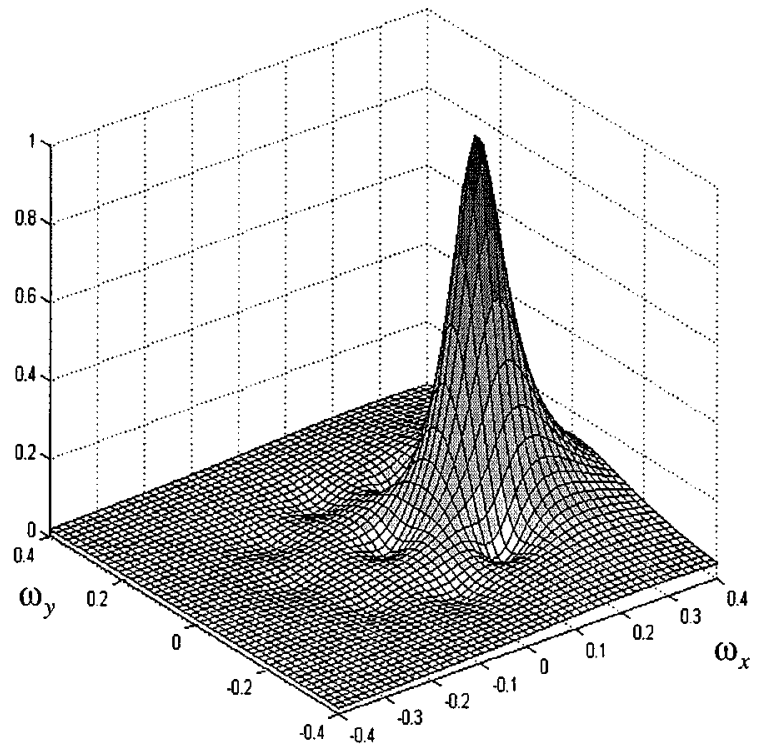

(a)

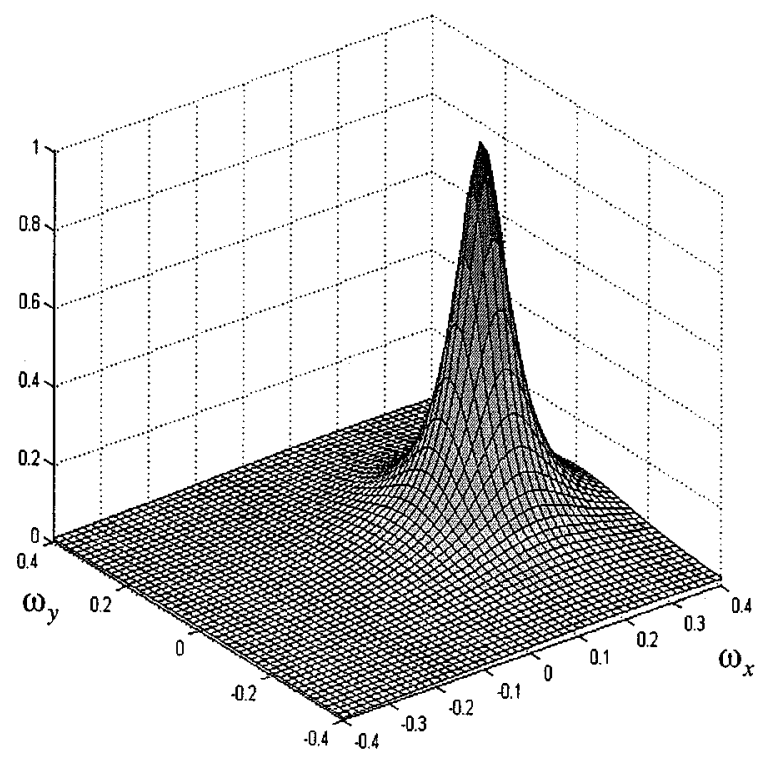

(b)

Fig. 5. Surface plots of the frequency responses of the coupled (a) and uncoupled (b) layers tuned to orientations $\Theta=0$ for the settings used in Fig. 4(a).

passband to the sector between $\theta_{k}-\pi / K$ and $\theta_{k}+\pi / K$. Thus, the orientation bandwidth increases as $Q$ decreases, but has a maximum value of $2 \pi / K$. The final layer introduces a null at DC in the frequency responses of the first $K$ layers. This improves the phase properties of the filters by making the real and imaginary parts of the impulse response better approximations to Hilbert pairs [9].

To illustrate, we computed the responses of a set of nine layers numerically. The first eight layers were tuned to spatial frequency magnitude $\Omega=\pi / 16$ and orientations $0, \pi / 4, \pi / 2,3 \pi / 4, \pi, 5 \pi / 4,3 \pi / 2$ and $7 \pi / 4$. The ninth layer was tuned to spatial dc. We considered the case where the layers had an uncoupled $Q=1$ and where the layers had an uncoupled $Q=0.1$. For comparison, we also computed the responses of uncoupled arrays tuned to have the same orientation bandwidth. This tuning is not the same as the uncoupled tuning of the coupled arrays, which is much broader.

Fig. 4 shows the frequency responses of the first eight orientation selective layers. The spatial-frequency magnitude bandwidth decreases as the uncoupled $Q$ increases. The cross section at constant orientation shows that this increase is primarily due to increased response to high frequencies. The layer tuned to dc constrains the passband to lie in the region $\omega>\Omega / 2$. The cross-section also illustrates that the increased spatial frequency bandwidth is accompanied by a decreased rejection of negative frequencies. Nulls in the frequency response at DC and at $-\Omega$ are introduced by competition from the layers tuned to dc and $\Theta=-\pi$. The cross-section at constant spatial frequency magnitude indicates that the orientation bandwidth is primarily determined by the locations of the competing orientations and less influenced by changes in the uncoupled $Q$. The competitive couplings' interconnections do not significantly affect the passband in comparison to a similarly tuned uncoupled layer, but improve the rejection of unwanted orientations by introducing nulls in the stopband. Fig. 5 shows surface plots of the frequency responses of the coupled and uncoupled layers tuned to orientation $\Theta=0$, for the same settings as used to generate the contour plots in Fig. 4(a). Note the nulls in the frequency response which are introduced by the competitive couplings.

Fig. 6 shows the imaginary parts of the impulse responses for the layer tuned to orientation $\Theta=\pi / 4$ for the four cases shown in Fig. 4. Due to the wider spatial frequency bandwidth, the impulse responses for the coupled system are more compact.

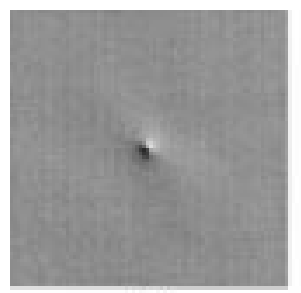

(a)

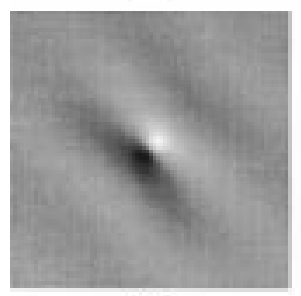

(c)

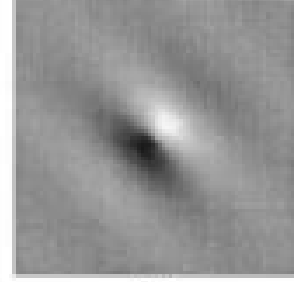

(b)

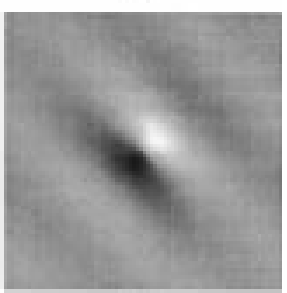

(d)
Fig. 6. Imaginary parts of the impulse responses of layers tuned to $\pi / 4$. (a) Response for the coupled system with uncoupled $Q=0.1$. (b) Response for an uncoupled layer tuned to the same orientation bandwidth in (a). (c) Response for the coupled system with uncoupled $Q=1$. (d) Response for an uncoupled layer tuned to the same orientation bandwidth in (c).

Fig. 7 compares the energy of the coupled and uncoupled filter outputs tuned to $\theta=0$ and $\theta=\pi / 4$ to a circular annulus. The filter energy is defined to be the sum of the squared real and imaginary parts of the response. The annulus has a Gaussian intensity radial cross section with $\sigma=1 / \Omega$ to match the tuned spatial frequency magnitude. The radius of the annulus was $10 \sigma$. Both coupled and uncoupled systems exhibit similar orientation tuning, but the coupled system has better rejection of undesired orientations.

Fig. 8 shows a polar plot representation of the filter outputs to sinusoidal gratings with spatial frequency $\Omega$, but varying orientations. For each trace, the angle of each point represents the input orientation and the distance from the origin represents the magnitude of the response. The sharper tuning by the coupled system is most noticeable in the real part of the response. 


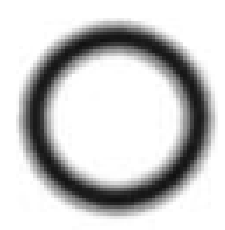

(a)

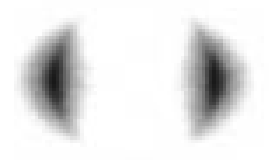

(b)

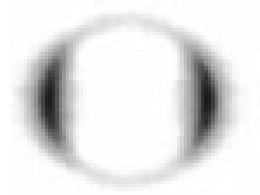

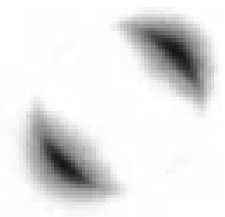

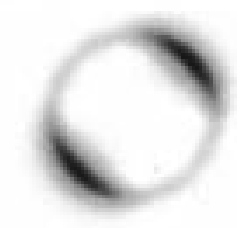

(c)

Fig. 7. The energy of the filter response of the layers tuned to orientations 0 and $\pi / 4$ for a circular annulus input. The response of the other orientation layers are similar up to a rotation. The coupled system had an uncoupled $Q=0.1$. Responses for $Q=1$ are similar. (a) The input image. (b) Responses of the coupled layers. (c) Responses of uncoupled layers with the same orientation bandwidth.

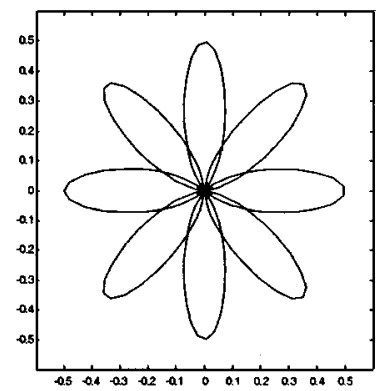

(a)

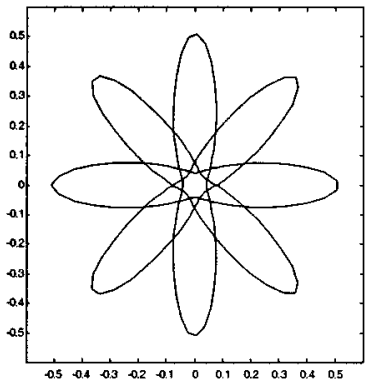

(c)

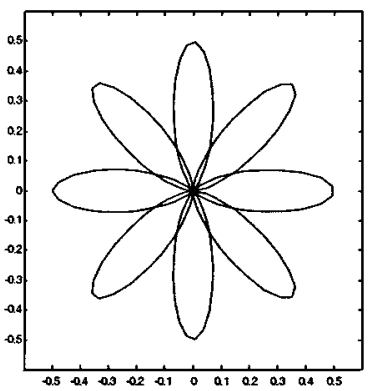

(b)

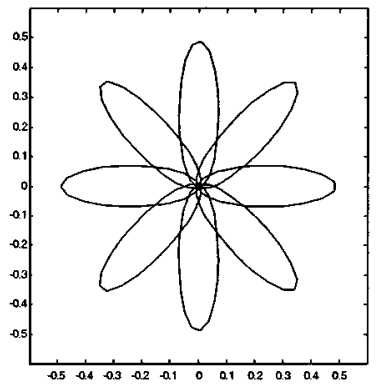

(d)
Fig. 8. Polar plots of the response of the real and imaginary parts of the filter outputs to sinusoidal gratings with frequency $\Omega=\pi / 16$ at varying orientations. (a) Real part of the response for the coupled system with $Q=1$. (b) Imaginary part of the response for the coupled system. (c) Real part of the response for uncoupled layers with the same orientation bandwidth. (d) Imaginary part of the response for uncoupled layers.

\section{CONCLUSION}

We have described a multilayer cellular neural network for orientation selective filtering which includes competitive couplings between layers tuned to different spatial frequencies. We have proven that this competition enhances spatial frequency tuning by introducing nulls in the stopband and constraints on the passband of the coupled layers. System level simulations on a nine layer system are used to illustrate these effects. However, this sharpening comes at the cost of increased system order and settling time.

Our ongoing work in this area seeks to implement these orientation selective circuits in CMOS VLSI. One of the advantages of this formulation is that the circuits performing the minimization are natural extensions of our previously designed circuits. In particular, because the intra-layer feedback templates $A_{k k}$ are identical in the coupled and uncoupled systems, circuits previously developed to implement these intra-layer connections for the uncoupled system can be used directly to implement the same interconnections in the coupled system.

Because of the limitations of the 2-D silicon substrate, we plan to split the processing among multiple chips. Each chip will implement a separate orientation selective layer and its associated intra-layer connections. The competitive inter-layer interconnections between the chips will be implemented via the address event representation (AER) communication protocol [10]. An important concern in this work is whether delays introduced by the AER coupling will significantly impact the performance of the coupled layers, e.g., the stability. Another alternative for implementation might be 3-D VLSI technology [11].

\section{ACKNOWLEDGMENT}

The authors would like to thank J. Chasnov for helpful discussions regarding this work.

\section{REFERENCES}

[1] L. O. Chua and T. Roska, "The CNN paradigm," IEEE Trans. Circuits Syst. I, vol. 40, pp. 147-156, Mar. 1993.

[2] B. E. Shi, "Gabor-type filtering in space and time with cellular neural networks," IEEE Trans. Circuits Syst. I, vol. 45, pp. 121-132, Feb. 1998.

[3] _ - "A low power orientation selective vision sensor," IEEE Trans. Circuits Syst. II, vol. 47, no. 5, pp. 435-440, May 2000.

[4] D. Gabor, "Theory of communication," J. IEE London, vol. 93, no. 3/26, pp. $429-457,1946$.

[5] R. Etienne-Cummings and J. Van der Spiegel, "Neuromorphic vision sensors," Sensors and Actuators A: Physical, vol. 56, no. 1/2, pp. 19-29, 1996.

[6] G. Cauwenberghs and J. Waskiewicz, "Focal-plane analog VLSI cellular implementation of the boundary contour system," IEEE Trans. Circuits Syst. I, vol. 46, pp. 327-334, Feb. 1999.

[7] T. Serrano-Gotarredona, A. G. Andreou, and B. Linares-Barranco, "A 2D image filtering architecture of real-time vision processing systems," in Proc. 7th. Int. Conf. on Microelectronics for Neural, Fuzzy and BioInspired Systems, Granada, Spain, Apr. 1999, pp. 415-422.

[8] J. G. Daugman, "Complete discrete 2-D Gabor transforms by neural networks for image analysis and compression," IEEE Trans. Acoust., Speech, Signal Processing, vol. 36, pp. 1169-1179, Jul. 1988.

[9] C.-J Westelius, H. Knutsson, J. Wiklund, and C.-F. Westin, "Phase-based disparity estimation," in Vision as Process, J. L. Crowley and H. I. Christensen, Eds. Berlin, Germany: Springer-Verlag, 1995, ch. 11, pp. $157-178$.

[10] K. A. Boahen, "Point-to-point connectivity between neuromorphic chips using address events," IEEE Trans. Circuits Syst. II, vol. 47, pp. 416-434, May 2000.

[11] H. Kurino, M. Nakagawa, K. W. Lee, T. Nakamura, Y. Yamada, K. T. Park, and M. Koyanagi, "Smart vision chip fabricated using three dimensional integration technology," in Advances in Neural Information Processing Systems, T. Leen, T. Dietterich, and V. Tresp, Eds. Cambridge, MA: MIT Press, 2001, vol. 13. 